EPJ Web of Conferences 38, 17011 (2012)

DOI: $10.1051 /$ epjconf/20123817011

C Owned by the authors, published by EDP Sciences, 2012

\title{
Mass-energy distribution of fragments in Langevin dynamics of fission induced by heavy ions
}

\author{
Yu. A. Anischenko ${ }^{1}$, D. V. Vanin ${ }^{2}$, and G. D. Adeev ${ }^{1}$ \\ 1 Omsk State University, Omsk, Russia \\ 2 Flerov Laboratory of Nuclear Reactions, Joint Institute for Nuclear Research, Dubna, Russia
}

\begin{abstract}
Four-dimensional Langevin equation was employed to calculate mass-energy distributions of fission fragments of highly excited compound nuclei. The research took into account not only three shape collective coordinates introduced on the basis of $\{c, h, \alpha\}$-parametrization but also orientation degree of freedom ( $K$-state $)-$ spin about the symmetry axis. Overdamped Langevin equation was used to describe the evolution of the $K$-state. Friction tensor was calculated using the "wall+window" model of the modified one-body dissipation mechanism with a reduction coefficient from the "wall" formula $k_{s}$. The calculations have been performed with $k_{s}=0.25$ and $k_{s}=1.0$. To learn more about the role of the dissipation effects the calculations have also been done with use of the chaoticity measure of nucleon movements in the nuclear shape configuration as $k_{s}$ parameter. Calculations were performed for the large number of compound nuclei with $Z^{2} / A$ parameter in the range $21 \leqslant Z^{2} / A \leqslant 44$. The goal was to study the mass-energy distributions not only for heavy nuclei but also for light nuclei close to the Businaro-Gallone point. Mass-energy distributions and variances of the mass fragments are well reproduced in the applied calculations for all considered compound nuclei. It was shown that inclusion of the $K$-state in the dynamical model produces considerable increase of the mass and energy variances. Inclusion of the chaoticity measure to the friction tensor provides a better agreement with the experiment results on mass variances.
\end{abstract}

\section{Introduction}

Mass-energy distributions of fission fragments are traditionally used as one of the main sources of information about the dynamics of the fission process and about the mechanism that governs the separation of a nucleus into fragments. Systematic experimental and theoretical investigations of mass-energy distributions were pioneered in the classic studies of Plasil and his coauthors [1] and Nix and Swiatecki [2]. In the past decades, extensive experimental investigations performed by research groups from Almaty and Dubna and devoted to exploring mass-energy distributions yielded a vast body of important information. The majority of those experimental studies were systematized and analyzed in the review articles quoted in [3-6].

A stochastic approach to fission dynamics on the basis of three-dimensional Langevin equations $[7,8]$ makes it possible to perform a comprehensive study of mass-energy distributions of fission fragments and mean multiplicities of prescission neutrons. The results of this systematic investigation were previously published in $[7,8]$. The formation of compound nuclei that have rather high excitation energies and high angular momenta is a feature peculiar to the fission process induced by heavy ions. On one hand, this circumstance suggests that the role of shell effects is insignificant in such reactions; on the other hand, it is indicative of the need for explicitly taking into account the orientation of nuclei in developing a model for fission induced by heavy ions. Unfortunately, the overwhelming majority of models of the fission process induced by heavy ions do not treat the orientation of nuclei as an independent collective coordinate. This entails, in addition to the impossibility of dynamically describing the angular distribu- tion of fragments, an erroneous estimation of mass-energy distribution of fission fragments, the mean multiplicity of prescission particles, the fission rate, and the mean fission time.

Eremenko and his coauthors $[9,10]$ were the first who proposed considering the evolution of the orientation degree of freedom of the nucleus involved ( $K$ mode - that is, the projection of the total angular momentum $I$ onto the symmetry axis of the nucleus) as an independent collective coordinate, relying on the Monte Carlo method implemented with the aid of an algorithm that simulates the Anderson-Kubo process. They were able to describe successfully the angular distributions of fission fragments and mean multiplicities of prescission neutrons for a number of fusion-fission reactions involving heavy ions. An alternative method for considering the evolution of the $K$ mode was proposed by Lestone [11] and by Lestone and McCalla [12]. They described the evolution of the coordinate $K$ in terms of the Langevin equation in the overdamped mode. The Langevin equation for the coordinate $K$ permits simulating the relaxation of $K$ states with allowance for instantaneous physical properties of the fissioning system, such as its temperature and moment of inertia, instead of treating the respective relaxation time as a free parameter $[9,10]$; moreover, it describes the evolution of all collective degrees of freedom of the nucleus within a unified conceptual framework. Thus, the Langevin dynamics of fission induced by heavy ions must include at least four collective coordinates-three for the evolution of the nuclear shape $[7,8]$ and one for the evolution of the $K$ mode [1116].

The four-dimensional dynamical model has been constructed on the basis of the three-dimensional model $[7,8]$. 
In the present study, we rely on four-dimensional Langevin dynamics [13-16], taking into account, in addition to three nuclear-shape coordinates considered traditionally, the evolution of the orientation degree of freedom. No systematic investigation of mass-energy distributions of fission fragments on the basis of Langevin dynamics with allowance for the evolution of the $K$ mode has been performed so far. In applying this model to studying mass-energy distributions of fragments of compound nuclei, we decided to use, in addition to constant values of $k_{s}=0.25$ and 1.0 , the the elongation-dependent reduction factor for the contribution from the wall formula, $k s=\mu\left(q_{1}\right)$, where, we recall, $q_{1}$ is the elongation parameter, which is the main fission coordinate. In order to calculate this dependence, we follow [1719], relying on the idea that the reduction factor for the contribution from the wall formula is intimately related to the measure of chaoticity of nucleons motion within a nucleus as this nucleus evolves from the ground state to separated shapes $[17,18]$. The explicit form of the function $\mu\left(q_{1}\right)$ was taken from [19].

Following [12], we choose the friction parameter of the $K$-mode to be constant equal to $0.077(\mathrm{MeV} \cdot \mathrm{zs})^{-1 / 2}$ and did not vary this quantity in the present study.

We would like to mention that in contrast to what was done in the earlier studies of our group that were reported in $[7,8]$, we did not go over here from a dynamical to a statistical description in simulating the process of compoundnucleus fission. This transition was performed earlier with aim of reducing The time of the calculations. With currently available computer facilities, we decided on dispensing with the transition to the statistical branch and described the fission process by using the Langevin approach exclusively. The main motivation of this choice was that the Kramers multidimensional formula, which underlies statistical calculations, does not lead to good agreement with the steady-state fission-rate level obtained from the dynamical calculations in [20].

The statistical model we used only to calculate multiplicities of light particles emitted from compound nucleus. As the compound nucleus evolves from the ground state to the scission point (along the Langevin trajectory), it may emit light prescission particles $(j=n, p, \alpha, \gamma)$; we took this into account by means of a Monte Carlo procedure [21]. After the evaporation of a prescission particle, we rescaled the temperature of the nucleus and all dimensional factors.

\section{Results and discussions}

\subsection{Influence of the $\mathrm{K}$ coordinate on the driving potential}

It should be noted that the driving force in an excited system is not simply the negative gradient of the bare potential $V(\mathbf{q})$, but should contain a thermodynamical correction [7, $8,22]$. In the present study the Helmholtz free energy $F(\mathbf{q})$ is the thermodynamical driving potential.

It is useful to discuss the static influence of the $K$ coordinate on the driving potential landscape before comparing the results of dynamical calculations with experimental data. The crucial changes of potential energy come from rotational energy. The latter increases the fission barrier height for the values $K \neq 0$ [12]. The example of the Helmholtz free energy potential along the fission path

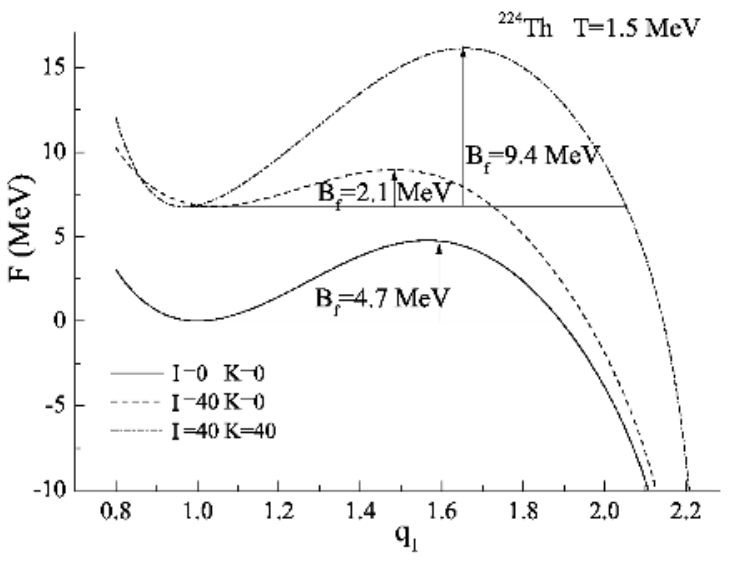

Fig. 1. The Helmholtz free energy along the mean fission trajectory for the ${ }^{224} \mathrm{Th}$ compound nucleus as the function of the elongation parameter $q_{1}$, and corresponding fission barriers $\left(B_{f}\right)$ for different combinations of $I$ and $K$ values.

for ${ }^{224} \mathrm{Th}$ is shown in figure 1 for different combinations of $I$ and $K$ values. A substantial increase of fission barrier could be obtained at large angular momenta, whenever $K$ is different from zero. Such increase of fission barrier will reduce the fission rate (increase the mean fission time) and increase the number of evaporated prescission particles. This effect is qualitatively equivalent to the increase of the dissipation strength in 3D calculations. Therefore, it is expected that in 4D calculations a lower value of dissipation strength will result in similar fission probabilities and prescission particles multiplicities as does a larger dissipation coefficient in 3D calculations.

The rotational energy decreases the stiffness (stability) of nuclei with respect to the mass asymmetry deformations $d^{2} F / d \eta^{2}$ for the values $K \neq 0$. Here $\eta=2\left(M_{1}-M_{2}\right) /\left(M_{1}+\right.$ $M_{2}$ ) is the mass-asymmetry coordinate, which was introduced by Strutinsky [23] and which is frequently used in analyzing relevant experimental data $[3,4,6]$. The $d^{2} F / d \eta^{2}$ values along the mean fission trajectory are shown in figure 2 for the nucleus ${ }^{224} \mathrm{Th}$. The stiffness of the nucleus with respect to the mass asymmetry $d^{2} F(K \neq 0) / d \eta^{2}$ is lower than $d^{2} F(K=0) / d \eta^{2}$ for all deformations with $q_{1}>$ 1.55 , which correspond to the descent from saddle to scission point. This lowering directly comes from the inclusion of $K$ coordinate, as $d^{2} F(I=0, K=0) / d \eta^{2}$ and $d^{2} F(I=40, K=0) / d \eta^{2}$ are substantially higher than $d^{2} F(I=40, K=40) / d \eta^{2}$. The difference between $d^{2} F(I=$ $0, K=0) / d \eta^{2}$ and $d^{2} F(I=40, K=40) / d \eta^{2}$ increases with deformation, and it reaches at the scission point around $10 \%$ for the compound nucleus ${ }^{248} \mathrm{Cf}$ and $12 \%$ for ${ }^{224} \mathrm{Th}$. The increase of angular momentum $I$ only slightly increases the stiffness of the nucleus with respect to mass asymmetry, whereas increase of $K$ decreases it more notably. This decreasing of compound nucleus stability with respect to mass asymmetry coordinate after inclusion of $K$ coordinate should make the mass distribution of fission fragments broader with respect to the $3 \mathrm{D}$ calculations, where $K=0$ is supposed during the fission process.

In the present analysis, we investigated the behavior of $d^{2} F(I, K) / d \eta^{2}$ at saddle and scission points for a wide set of beta-stable nuclei and results are presented in figure 3 . The main changes of stiffness at both saddle and scission points $\left(d^{2} F(I, K) / d \eta^{2}\right)_{\text {sd,sc }}$ are caused by the inclusion of 


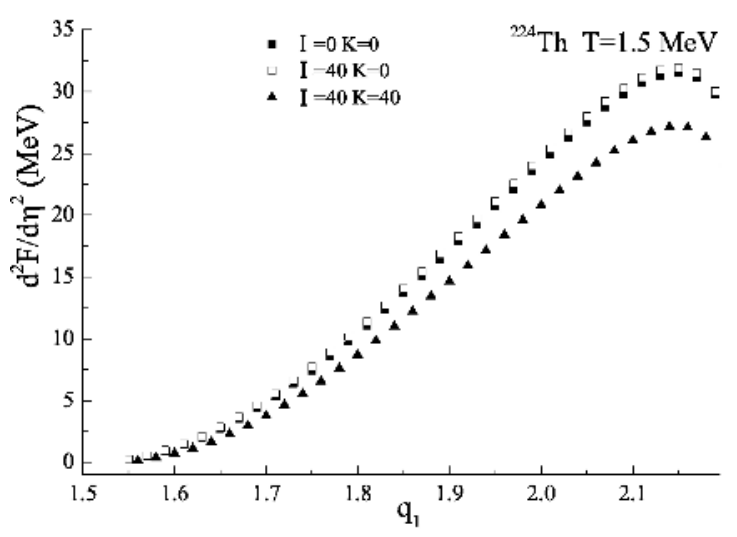

Fig. 2. Stiffness of the ${ }^{224} \mathrm{Th}$ compound nucleus with respect to the mass asymmetry coordinate $\eta$ along the mean fission trajectory as a function of the elongation parameter $q_{1}$. Different combinations of $I$ and $K$ are considered as indicated.

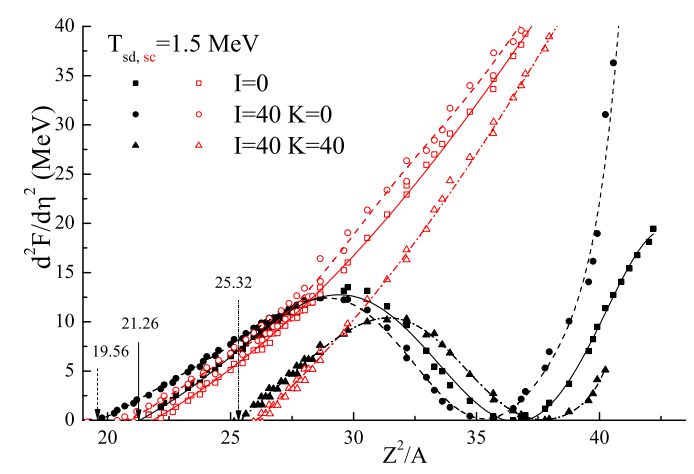

Fig. 3. The stiffness of the beta-stable nuclei with respect to mass asymmetry coordinate at the saddle (black full symbols and curves) and scission (red open symbols and curves) points, $d^{2} F / d \eta^{2}$ (in $\mathrm{MeV}$ ), for nuclei along the beta-stability line at temperature $T=1.5 \mathrm{MeV}$. The solid curves correspond to the polynomial approximation for the calculated data for the non-rotating nucleus $(I=0)$. Dashed and dash-dotted curves are polynomial approximations for the $I=40$ and $K=0$ and $K=I=40$ cases respectively. The arrows with numbers indicate the corresponding Businaro- Gallone point for each pair of $I$ and $K$.

$K$ coordinate for all considered nuclei in the range $20<$ $Z^{2} / A<42$. The increase of $K$ qualitatively results in a parallel shift of the $\left(d^{2} F(I, K) / d \eta^{2}\right)_{\text {sd,sc }}$ curves presented in figure 3 towards larger $Z^{2} / A$ values. Hence, the BusinaroGallone point moves from approximately $Z^{2} / A \simeq 20$ for the case of $K=0$ to the $Z^{2} / A \simeq 25$ for the case of $I=$ $K=40$. Another sizeable effect is the substantial decrease of mass asymmetry stiffness $\left(d^{2} F(I, K) / d \eta^{2}\right)_{\text {sd }}$ at saddle point for the heavy nuclei with $Z^{2} / A \simeq 40$. The value of $\left(d^{2} F(I=40, K=40) / d \eta^{2}\right)_{\text {sd }}$ is around 5 times smaller than $\left(d^{2} F(I=40, K=0) / d \eta^{2}\right)_{\text {sd. }}$. Therefore, for the heavy compound nuclei considered in the present paper the inclusion of $K$ coordinate should increase the width of mass distribution.

\subsection{The mass-energy distributions of fission fragments and analysis of results}

The method to calculate mass-energy distributions of fission fragments is described in our earlier publications $[7,8$,
Table 1. Results of theoretical calculations for the variance of the mass distribution of fission fragments along with experimental data. The theoretical calculations for $\sigma_{M}^{2}$ were performed at $k_{s}=$ $\mu\left(q_{1}\right)$; the experimental data for $\sigma_{M}^{2(\text { expt })}$ were borrowed from [4, 5]; the variances of the mass distribution of fission fragments, $\sigma_{M}^{2}$, are given in $(a m u)^{2}$ units; and the $E^{*}$ values are given in $\mathrm{MeV}$ units

\begin{tabular}{llll}
\hline Reaction & $E^{*}$ & $\sigma_{M}^{2}$ (expt) & $\sigma_{M}^{2}$ \\
\hline${ }^{93} \mathrm{Nb}+{ }^{12} \mathrm{C} \longrightarrow{ }^{105} \mathrm{Ag}$ & 121 & 667 & $605 \pm 154$ \\
${ }^{28} \mathrm{Si}+{ }^{89} \mathrm{Y} \longrightarrow{ }^{117} \mathrm{I}$ & 83.82 & 306 & $274 \pm 20$ \\
${ }^{78} \mathrm{Kr}+{ }^{40} \mathrm{Ca} \longrightarrow{ }^{118} \mathrm{Ba}$ & 98.8 & - & $274 \pm 19$ \\
${ }^{18} \mathrm{O}+{ }^{154} \mathrm{Sm} \longrightarrow{ }^{172} \mathrm{Yb}$ & 127.75 & 228 & $258 \pm 13$ \\
${ }^{16} \mathrm{O}+{ }^{170} \mathrm{Er} \longrightarrow{ }^{186} \mathrm{Os}$ & 129 & 242 & $265 \pm 22$ \\
${ }^{4} \mathrm{He}+{ }^{209} \mathrm{Bi} \longrightarrow{ }^{213} \mathrm{At}$ & 69.2 & 160 & $226 \pm 15$ \\
${ }^{18} \mathrm{O}+{ }^{197} \mathrm{Au} \longrightarrow{ }^{215} \mathrm{Fr}$ & 112.9 & 272 & $334 \pm 5$ \\
${ }^{16} \mathrm{O}+{ }^{208} \mathrm{~Pb} \longrightarrow{ }^{224} \mathrm{Th}$ & 53.8 & 224 & $244 \pm 9$ \\
${ }^{12} \mathrm{C}+{ }^{232} \mathrm{Th} \longrightarrow{ }^{244} \mathrm{Cm}$ & 69.3 & 366 & $385 \pm 8$ \\
${ }^{20} \mathrm{Ne}+{ }^{232} \mathrm{Th} \longrightarrow{ }^{252} \mathrm{Fm}$ & 93.4 & 565 & $516 \pm 6$ \\
${ }^{18} \mathrm{O}+{ }^{246} \mathrm{Cm} \longrightarrow{ }^{264} \mathrm{Rf}$ & 52.3 & 435 & $497 \pm 5$ \\
${ }^{16} \mathrm{O}+{ }^{249} \mathrm{Cf} \longrightarrow{ }^{265} \mathrm{Sg}$ & 89.4 & 664 & $621 \pm 6$ \\
${ }^{20} \mathrm{Ne}+{ }^{249} \mathrm{Cf} \longrightarrow{ }^{269} \mathrm{Hs}$ & 50.7 & 725 & $554 \pm 12$ \\
\hline
\end{tabular}

22]. The wide set of experimental data available for the reactions has been analyzed using the new four-dimensional dynamical model. The dependence of the calculated variances of mass distributions of fission fragments, $\sigma_{M}^{2}$, on the parameter $Z^{2} / A$ is presented in table 1 and figure 4 along with experimental data. The growth of the variance of the mass distribution with the parameter $Z^{2} / A$ was reproduced for nuclei characterized by values of this parameter in the region of $Z^{2} / A>33$. In the region of nuclei for which $Z^{2} / A=21-33$, the growth of $\sigma_{M}^{2}$ as the parameter $Z^{2} / A$ decreases is also reproduced. Agreement with experimental data in the case of $k_{s}=0.25$ or in the case of $k_{s}=\mu\left(q_{1}\right)$ is not only qualitative but also quantitative (the deviation from experimental data is not more than $30 \%$ ). For the reaction leading to the production of the compound nucleus ${ }^{105} \mathrm{Ag}$, the values of the variances are anomalous, which is indicative of the proximity of this nucleus to the BusinaroGallone point. In [7,8], the calculations of $\sigma_{M}^{2}$ were performed on the basis of the three-dimensional model (that is, without allowance for the $K$ mode). Those calculations revealed that, by and large, the three-dimensional model describes well the mass distributions at $k_{s}=0.25$, but that values obtained for the variance of the mass distribution of fission fragments were underestimated in relation to experimental data for heavy nuclei. From our present calculations, it follows that the inclusion of the $K$ mode improves the agreement with experimental data, increasing the variances of masses in relation to the results that the three-dimensional model yields for heavy nuclei. The effect of an increase in variances for heavy nuclei upon going over from the three to the four-dimensional model is due to a decrease in the stiffness of the fissile nucleus against mass-asymmetric deformations over the segment of descent from the saddle to the scission point. Figure 2 demonstrates how the dependence of the stiffness $\partial^{2} F / \partial \eta^{2}$ on the coordinate $q_{1}$ changes upon taking into account the $K$ mode. From figure 2, one can see that the inclusion of the $K$ mode leads to a decrease in the values of $\partial^{2} F / \partial \eta^{2}$ over the segment of descent from the saddle to the scission point for heavy nuclei, and this causes an increase in 


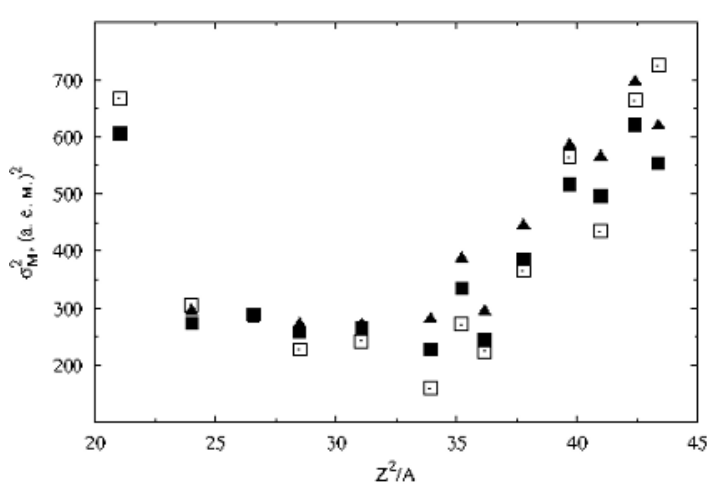

Fig. 4. Variance of the mass distribution of fission fragments $\sigma_{M}^{2}$, as a function of the parameter $Z^{2} / A$ for nuclei in the beta-stability valley: (open boxes) experimental data from $[4,5]$, (closed triangles) results of theoretical calculations at $k_{s}=0.25$, and (closed boxes) $k_{s}=\mu\left(q_{1}\right)$.

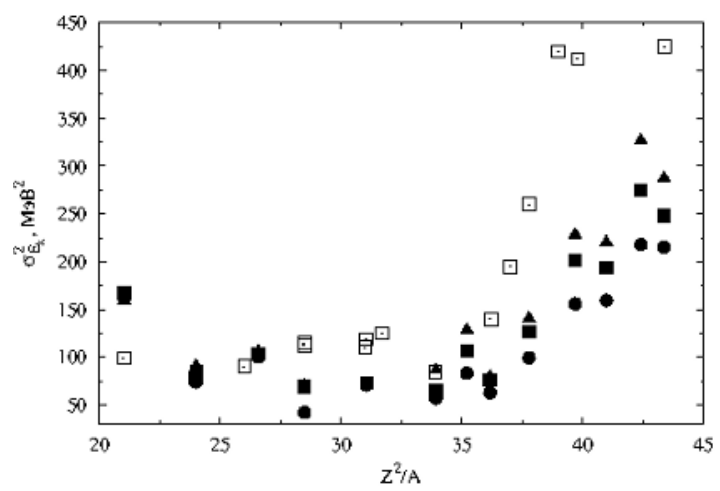

Fig. 5. Variance of the energy distribution of fission fragments $\sigma_{E_{k}}^{2}$, as a function of the parameter $Z^{2} / A$ for nuclei in the beta-stability valley according to (open boxes) experimental data from $[4,5]$ and according to the results of our theoretical calculations for the cases of(closed triangles) $k_{s}=0.25$, (closed boxes) $k_{s}=\mu\left(q_{1}\right)$, and (closed circles) $k_{s}=1.0$.

the values of the variances. The $Z^{2} / A$ dependence of the calculated variances of the energy distribution of fission fragments, $\sigma_{E k}$, is presented in figure 5 for various values of $k_{s}$. Good qualitative agreement with experimental data is achieved in our calculations, but the variances of energies are strongly underestimated for reactions involving the production of heavy compound nuclei. The deviation from experimental data is 30 to $100 \%$ In all probability, additional coordinates responsible for the degree of deformation of fission fragments must be introduced in order to improve the agreement with experimental data.

\section{Conclusions}

The four-dimensional dynamical model has been developed on the basis of the three-dimensional model by adding the orientation degree of freedom $\mathrm{K}$ to the three collective coordinates which describe the shape of the fissioning nucleus. It was found that the $\mathrm{K}$ degree of freedom not only increases fission barrier height, but also changes the stiffness of the nucleus with respect to mass asymmetry coordinate.
The prescission neutron multiplicities, fission fragment mass-energy distribution parameters could be reasonably reproduced for heavy nuclei with the dissipation coefficients $k_{s}=\mu\left(q_{1}\right)$ and $\gamma_{K} \simeq 0.077(\mathrm{MeV} \cdot \mathrm{zs})^{-1 / 2}$ in contrast with three-dimensional calculations, where a self-consistent description of all observables with the same $k_{s}$ value was impossible for heavy nuclei. Careful accounting of the influence of the $K$ coordinate on the potential energy surface and on the dynamics of shape coordinates allows one to get a consistent picture of the fission process in fourdimensional Langevin calculations.

\section{References}

1. F. Plasil, D.S. Burnett, H.C. Britt and S.G. Thompson, Rhys. Rev. 142, 696 (1966)

2. J.R. Nix and W.J. Swiatecki, Nucl. Phys. A 71, 1 (1965) 1; J.R.Nix, Nucl. Phys. A 130, 241 (1969)

3. G.G. Chubaryan, M.G. Itkis and S.M. Lukyanov, et al., Phys. At. Nucl. 56, 286 (1993)

4. M.G. Itkis, Yu.A. Muzychka, Yu.Ts. Oganessian, et al., Phys. At. Nucl. 58, 2026 (1995)

5. A.Ya. Rusanov, M.G. Itkis and V.N. Okolovich, Phys. At. Nucl. 60, 683 (1997)

6. M.G. Itkis and A.Ya. Rusanov, Phys. Part. Nucl. 29, 160 (1998)

7. P.N. Nadtochy, G.D. Adeev and A.V. Karpov, Phys. Rev. C 65, 064615 (2002); A.V. Karpov, P.N. Nadtochy, D.V. Vanin, G.D. Adeev, Phys. Rev. C 63, 054610 (2001)

8. G.D. Adeev, A.V. Karpov, P.N. Nadtochy, and D.V. Vanin, Phys. Part. Nucl. 36, 378 (2005)

9. V.A. Drozdov, D.O. Eremenko, O.V. Fotina, et al., AIP Conf. Proc. 704, 130 (2004)

10. D.O. Eremenko et al., Phys. At. Nucl. 69, 1423 (2006)

11. J.P. Lestone, Phys. Rev. C 59, 1540 (1999)

12. J.P. Lestone and S.G. McCalla, Phys. Rev. C 79, 044611 (2009)

13. A.E. Gegechkori and G.D. Adeev, Phys. At. Nucl. 74, $1(2011)$

14. Yu.A. Anischenko, A.E. Gegechkori, G.D. Adeev, Phys. At. Nucl. 74, 341 (2011)

15. Yu.A. Anischenko and G.D. Adeev, Phys. At. Nucl. 75, 933 (2012)

16. P.N. Nadtochy, E.G. Ryabov, A.E. Gegechkori, Yu.A. Anischenko, G.D. Adeev, Phys. Rev. C 85, 064619 (2012)

17. S. Pal and T. Mukhopadhyay, Phys. Rev. C 57, 210 (1998)

18. J. Blocki, F. Brut, T. Srokowski, and W.J. Swiatecki, Nucl. Phys. A 545, 511 (1992)

19. G. Chaudhuri and S. Pal, Phys. Rev. C 63, 064603 (2001)

20. Yu.A. Anischenko, A.E. Gegechkori, P.N. Nadtochy, and G.D. Adeev, Phys. At. Nucl. 72, 1992 (2009)

21. M.D. Mavlitov, P. Fröbrich, and I.I. Gontchar, Z. Phys. A 342, 195 (1992)

22. E.G. Ryabov, A.V. Karpov, P.N. Nadtochy, G.D. Adeev, Phys. Rev. C 78, 044614 (2008)

23. V.M. Strutinsky, Zh. Eksp. Teor. Fiz. 45, 1891 (1963) 